\title{
Stellar metallicity distributions in local dwarf spheroidal galaxies: a comparison between model and observations
}

\author{
Gustavo A. Lanfranchi ${ }^{1}$ and Francesca Matteucci ${ }^{2}$ \\ ${ }^{1}$ Núcleo de Astrofísica Teórica, Universidade Cruzeiro do Sul, \\ R. Galvão Bueno 868, Liberdade, 01506-000, São Paulo, SP, Brazil \\ email: gustavo.lanfranchi@cruzeirodosul.edu.br \\ ${ }^{2}$ Dipartimento di Astronomia-Universitá di Trieste, \\ Via G. B. Tiepolo 11, 34131 Trieste, Italy
}

\begin{abstract}
We analyze the main populations of 6 local Dwarf Spheroidal Galaxies by comparing the observed stellar metallicity distributions with the predictions of chemical evolution models. The predicted metallicity distributions of stars generally exhibit a low range of metallicities, a peak below the one of the MW disc in the solar neighborhood (but similar to the Halo), and a sharp decrease at higher metallicities in agreement with observations. The position of the peak is related to the low star formation rates adopted whereas the sharp decrease is a consequence of the occurrence of strong galactic winds. In the low metallicity tail of the distributions we predict a low number of stars in agreement with observations.
\end{abstract}

Keywords. stars: abundances, galaxies: dwarf, Local Group

\section{The model}

We use the chemical evolution model for Dwarf Spheroidal (dSph) galaxies as described in Lanfranchi \& Matteucci $(2003,2004)$. Each galaxy model is specified by the prescriptions of the star formation $(\mathrm{SF})$ and by the galactic wind (GW) efficiency chosen to reproduce the main features of these galaxies $([\alpha / \mathrm{Fe}],[\mathrm{Fe}-\mathrm{peak} / \mathrm{Fe}],[\mathrm{s}-\mathrm{r} / \mathrm{Fe}]$, total mass, gas mass). The star formation histories (SFH) are constrained by the analyzes of color-magnitude diagrams which suggests a diversity and complexity of the SFH among the galaxies. The main characteristics of the models are the following: one zone with instantaneous and complete mixing of gas inside this zone; the stellar lifetimes are taken into account; the SFR is proportional to the gas mass and given by its efficiency $(\nu)$; the wind efficiency $\left(w_{i}\right)$ is proportional to the SFR. In the nucleosynthesis prescriptions we adopted the yields of van den Hoeck \& Groenewegen (1997) for low and intermediate mass stars, Nomoto et al. (1997) for type Ia supernovae and Woosley \& Weaver (1995) for massive stars.

\section{The stellar metallicity distributions}

We compared the predictions of the models to the observed SMDs of six dSph galaxies of the Local Group. For Carina, Leo1, Leo 2, and Sculptor the metallicity is represented by $[\mathrm{Fe} / \mathrm{H}]$ inferred from the CaT line (Koch et al. 2006, 2007a, 2007b, Tolstoy et al. 2004, Lanfranchi, Matteucci \& Cescutti 2006) whereas metallicities derived from the colors of RGB stars, were used in Draco and Ursa Minor (Bellazzini et al. 2002, Lanfranchi \& Matteucci 2007). The general comparison allows one to characterize the main stellar population of each galaxy and to draw a scenario for their formation and evolution based 


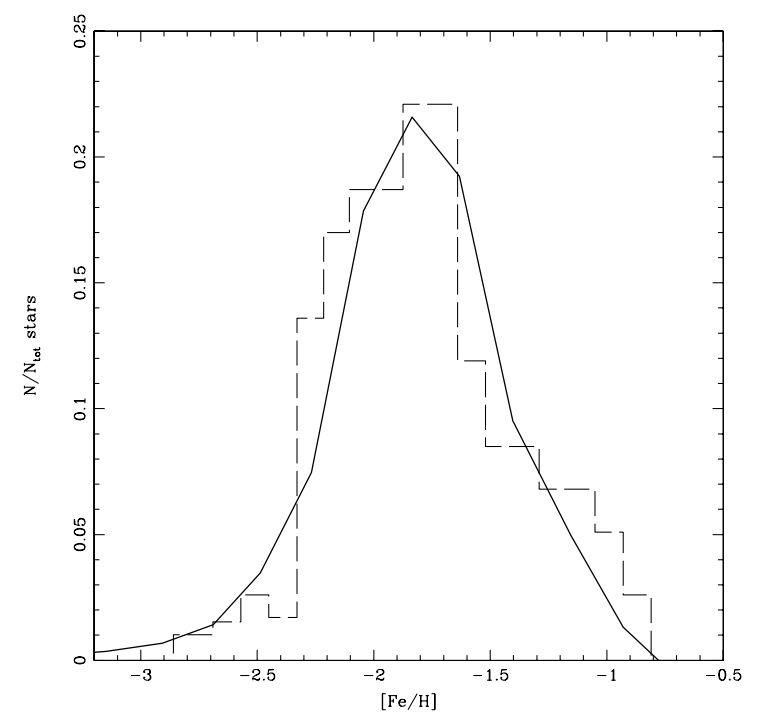

Figure 1. The SMD predicted by the model of Sculptor (solid thick line) compared to observations (dashed line).

in specific features of the SMDs, such as the low and high-metallicity tails and the position of the peak. These features are strongly influenced by the adopted SF and GW efficiencies. A low SF efficiency explains the low number of metal-poor stars and the position of the peak of the distribution, which is also fitted by a high GW efficiency. The very efficient wind is also responsible for the sharp decrease observed in the high-metallicity tail of the SMDs. The six dSph galaxies exhibit similar features in their stellar populations: a relative low number of metal-poor stars $([\mathrm{Fe} / \mathrm{H}]<-2.5 \mathrm{dex})$, a narrow range in metallicities (1.5 to $2.0 \mathrm{dex}$ ), a main stellar population with a low $[\mathrm{Fe} / \mathrm{H}](\sim-1.8-$ $1.5 \mathrm{dex}$ ), and a sharp decrease in the number of stars with metallicities higher than the one of the peak of the SMD $([\mathrm{Fe} / \mathrm{H}]>-1.0 \mathrm{dex})$ (see the case of Sculptor in Figure 1). Even though the SFHs differ from one system to another, all these galaxies should exhibit a common characteristic in order to reproduce the observed SMDs: the SFR is low (with a low efficiency) and and intense galactic wind (with a rate few times the SFR) took place, removing a large fraction of the ISM gas, decreasing substantially the SFR. These two facts coupled are the responsible for the main features observed in the SMD.

\section{References}

Bellazzini, M., et al., 2002, AJ, 124, 3222

Lanfranchi, G. \& Matteucci, F., 2003, MNRAS, 345, 71

Lanfranchi, G. \& Matteucci, F., 2004, MNRAS, 351, 1338

Lanfranchi, G., Matteucci, F., \& Cescutti, 2006, A\&A, 453, 67

Nomoto, K., et al., 1997, Nucl. Phys. A, 616, 79

Koch, A., Grebel, E. K., Wyse, R. F. G., et al., 2006, AJ, 131, 895

Koch, A., Grebel, E. K., Kleyna, J. T., et al., 2007a, AJ, 133,270

Koch A., Wilkenson M. I., Kleyna J. T., et al., 2007b, ApJ, 657, 241

Tolstoy, E., Irwin, M. J., Helmi, A., et al. 2004, ApJ, 617, L119

van den Hoeck, L. B. \& Groenwegen, M. A. T., 1997, A\&AS, 123, 305

Woosley, S. E. \& Weaver, T. A., 1995, ApJS, 101, 181 